\title{
PKC/Nedd4-2 Signaling Pathway Regulates the Cell Surface Expression of Drug Transporter hOAT1
}

\author{
Da Xu, Jinghui Zhang, Qiang Zhang, Yunzhou Fan, ${ }^{1}$ Chenchang Liu, ${ }^{1}$ and Guofeng You \\ Department of Pharmaceutics, Rutgers University, Piscataway, New Jersey
}

Received March 9, 2017; accepted May 26, 2017

\begin{abstract}
Human organic anion transporter-1 (hOAT1) regulates the absorption, distribution, and excretion of a wide range of clinically important drugs. Our previous work demonstrated that hOAT1 is a dynamic membrane transporter, constitutively internalizing from and recycling back to the cell plasma membrane. Short-term activation ( $<30$ minutes) of protein kinase $C$ (PKC) promotes the attachment of a lysine 48-linked polyubiquitin chain to hOAT1, a process catalyzed by ubiquitin ligase neural precursor cell expressed developmentally down-regulated 4-2 (Nedd4-2). The ubiquitination of hOAT1 then triggers an accelerated endocytosis of the transporter from plasma membrane, which results in reduced hOAT1 expression at the cell surface and decreased hOAT1 transport activity. In the present study, we investigated the long-term effect of PKC on hOAT1. We showed that long-term activation $>2$
\end{abstract}

hours) of PKC significantly enhanced hOAT1 degradation, and such action was partially blocked by ubiquitin mutant Ub-K48R, which has its lysine (K) 48 mutated to arginine $(R)$ and is incapable of forming a K48-linked polyubiquitin chain. The ubiquitin ligase Nedd4-2 was also found to augment hOAT1 degradation. These results suggest that PKC-regulated and Nedd4-2-catalyzed attachment of a lysine 48-linked polyubiquitin chain to hOAT1 is important for hOAT1 stability. We further showed through coimmunoprecipitation experiments that there was a direct association between hOAT1 and Nedd4-2, and such interaction was weakened when the WW3 and WW4 domains of the ligase were mutated. Mutating WW3 and WW4 domains of the ligase also impaired its ability to ubiquitinate hOAT1. Therefore, WW3 and WW4 domains of Nedd4-2 are critical for its association with and modulation of the transporter.

\section{Introduction}

Human organic anion transporter-1 (hOAT1) is localized at the basolateral membrane of the renal proximal tubule cells. It regulates the excretion of a wide range of environmental toxins and clinical drugs, including anticancer drugs, antiviral agents, diuretics, antibiotics, antihypertension drugs, and anti-inflammatories (You, 2002; Terada and Inui, 2007; Ahn and Nigam, 2009; Pelis and Wright, 2011; Wang and Sweet, 2013). As a cell membrane transporter, the transport activity of hOAT1 is critically dependent on its expression level at the plasma membrane. Our previous work demonstrated (Zhang et al., 2008) that hOAT1 is a dynamic membrane transporter, constitutively internalizing from and recycling back to the cell surface. Short-term activation ( $<30$ minutes) of protein kinase $\mathrm{C}(\mathrm{PKC})$ promotes the attachment of a lysine 48-linked polyubiquitin chain to hOAT1, a process catalyzed by ubiquitin ligase neural precursor cell expressed developmentally downregulated 4-2 (Nedd4-2) (Zhang et al., 2013; Xu et al., 2016b). The Ubiquitination of hOAT1 then triggers an accelerated endocytosis of the transporter from the plasma membrane to intracellular endosomes, which results in decreased hOAT1 expression at the plasma membrane and decreased hOAT1 transport activity.

Recently, the post-translational modification by ubiquitin conjugation has become the major mechanism for regulating several membrane

This work was supported by the National Institutes of Health National Institute of General Medical Sciences [Grant R01-GM079123] (to G.Y.).

${ }^{1}$ Y.F. and C.L. contributed equally to this work.

https://doi.org/10.1124/dmd.117.075861. proteins in their internalization, intracellular sorting, and turnover rate (Staub and Rotin, 2006; Miranda and Sorkin, 2007). Ubiquitin, a highly conserved 8-kDa protein, forms a peptide bond between its glycine and lysine residues of the target protein. The ubiquitin conjugation can be either a single ubiquitin or a chain of ubiquitin proteins. The formation of a polyubiquitin chain occurs through the seven lysine residues of ubiquitin itself including K6, K11, K27, K29, K33, K48, and K63. Therefore, the ubiquitin conjugation can be classified as three types: monoubiquitination (attachment of one ubiquitin to one lysine on the target protein), multiubiquitination (attachment of several ubiquitins to several lysines on the target protein), or polyubiquitination (attachment of polyubiquitin chain(s) to one or more lysine on the target protein). Through mass spectrometry analysis, our laboratory recently demonstrated that PKC-promoted conjugation of ubiquitin to hOAT1 is the attachment of a lysine-48-linked polyubiquitin chain to the transporter (Zhang et al., 2013).

Nedd4-2, a member of the Nedd4 family of HECT ubiquitin ligases, catalyzes the ubiquitination of various mammalian transporters and channels (Snyder et al., 2004; Sorkina et al., 2006; Vina-Vilaseca and Sorkin, 2010; García-Tardón et al., 2012). Structurally, Nedd4 family proteins contain a catalytic HECT domain, $\mathrm{C} 2\left(\mathrm{Ca}^{2+} /\right.$ lipid binding $)$ domain, and 2-4 WW domains. The HECT domain at the C-terminus contains ubiquitin ligase activity. WW domains are involved in recognition and interaction with target proteins, while the $\mathrm{C} 2$ domain carries the membrane targeting function.

Despite significant progress made in our laboratory in the understanding the short-term regulation of hOAT1 by PKC, the longterm effect of PKC on hOAT1 has not been explored. In the current

ABBREVIATIONS: hOAT1, human organic anion transporter-1; Nedd4-2, neural precursor cell expressed developmentally down-regulated 4-2; PKC, protein kinase C; PMA, phorbol 12-myristate 13-acetate. 
study, we present evidence showing that Nedd4-2-catalyzed conjugation of a lysine 48-linked polyubiquitin chain to hOAT1 plays a significant role in the long-term PKC regulation of hOAT1 stability, and that the WW3 and WW4 domains of Nedd4-2 are critical for its association with and modulation of the transporter.

\section{Materials and Methods}

Materials. COS-7 cells were purchased from ATCC (Manassas, VA). $\left[{ }^{3} \mathrm{H}\right]-$ labeled $p$-aminohippuric acid was purchased from PerkinElmer (Waltham, MA). Membrane-impermeable biotinylation reagent sulfo-NHS-SS-biotin, streptavidinagarose beads, and protein G-agarose beads were purchased from Pierce (Rockford, IL). cDNA for human Nedd4-2 was generously provided by Dr. Peter M. Snyder (College of Medicine, University of Iowa, Iowa City, IA). HA-tagged ubiquitin mutant HA-Ub-K48R was generously provided by Dr. Cam Patterson (Carolina Cardiovascular Biology Center, University of North Carolina, Chapel Hill, NC). Mouse anti-myc antibody and anti- $\beta$-actin were purchased from Roche (Indianapolis, IN). Mouse anti-ubiquitin antibody P4D1 was purchased from Santa Cruz (Santa Cruz, CA). Mouse anti-FLAG (M2) antibody was purchased from Sigma-Aldrich (St. Louis, MO). Mouse anti-E-cadherin (M168) antibody was purchased from Abcam (Cambridge, MA). PKC activator phorbol 12-myristate 13-acetate (PMA) and all other reagents were purchased from Sigma-Aldrich.

Cell Culture and Transfection. hOAT1-expressing COS-7 cells were maintained in Dulbecco's modified Eagle's medium at $37^{\circ} \mathrm{C}$ in $5 \% \mathrm{CO}_{2}$. The medium contained $10 \%$ fetal bovine serum. hOAT1 was tagged with an epitope Myc to its carboxyl terminus for immunodetection of the transporter. Lipofectamine 2000 (Invitrogen, Carlsbad, CA) was used for the transfection of the cDNA

A hOAT1 expression at the cell surface

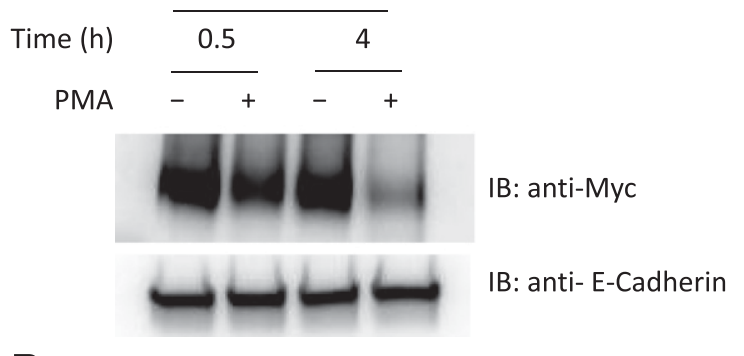

B

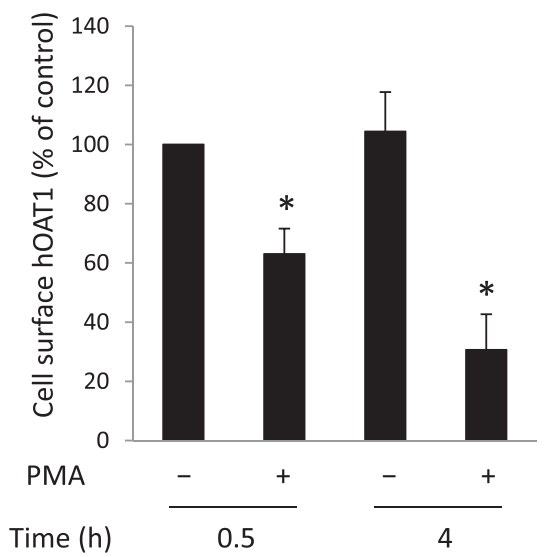

plasmids following the manufacturer's instructions. Forty-eight hours after transfection cells were harvested for further experiments.

\section{Site-Directed Mutagenesis}

Ubiquitin lysine 48 mutant (K48R) and Nedd4-2 WW domain mutants were generated in our laboratory using a QuickChange site-directed mutagenesis kit (Agilent Technologies, Santa Clara, CA), followed by the confirmation of the mutant sequences using the dideoxy chain termination method.

Measurement of hOAT1-Mediated Transport. The uptake solution consisted of phosphate-buffered saline/ $\mathrm{Ca}^{2+} / \mathrm{Mg}^{2+}(137 \mathrm{mM} \mathrm{NaCl}, 2.7 \mathrm{mM} \mathrm{KCl}$, $4.3 \mathrm{mM} \mathrm{Na}_{2} \mathrm{HPO} 4,1.4 \mathrm{mM} \mathrm{KH}_{2} \mathrm{PO} 4,0.1 \mathrm{mM} \mathrm{CaCl}_{2}$, and $1 \mathrm{mM} \mathrm{MgCl}_{2}, \mathrm{pH} 7.3$, and $20 \mu \mathrm{M}\left[{ }^{3} \mathrm{H}\right] p$-aminohippuric acid). The uptake solution was added to cells for indicated periods of time, and the uptake was terminated by removing the uptake solution and washing the cells with ice-cold phosphate-buffered saline solution. The cells were then solubilized in $0.2 \mathrm{~N} \mathrm{NaOH}$, neutralized in $0.2 \mathrm{~N} \mathrm{HCl}$, and subjected to liquid scintillation counting.

Cell Surface Biotinylation. The amount of hOAT1 at the plasma membrane was examined using the membrane-impermeable biotinylation reagent, sulfoNHS-SS-biotin. Cells were incubated with NHS-SS-biotin for 20 minutes twice on ice. Afterward, cells were washed with phosphate-buffered saline $/ \mathrm{Ca}^{2+} / \mathrm{Mg}^{2+}$ containing $100 \mathrm{mM}$ glycine to quench the unreacted sulfo-NHS-SS-biotin. The cells were then lysed in lysis buffer $(10 \mathrm{mM}$ Tris/ $\mathrm{HCl}, 150 \mathrm{mM} \mathrm{NaCl}, 1 \mathrm{mM}$ EDTA, $0.1 \%$ SDS, $1 \%$ Triton X-100 with $1 / 100$ protease inhibitor mixture and $20 \mathrm{mM} \mathrm{N}$-ethylmaleimide). The cell lysates were subjected to centrifugation at $16,000 \mathrm{~g}$ at $4^{\circ} \mathrm{C}$, followed by addition of streptavidin-agarose beads to the supernatant to isolate plasma membrane proteins. The surface proteins were then separated on SDS-PAGE, followed by immunoblotting using an anti-myc antibody to detect myc-tagged hOAT1.

\section{C hOAT1 expression in total cell lysates}

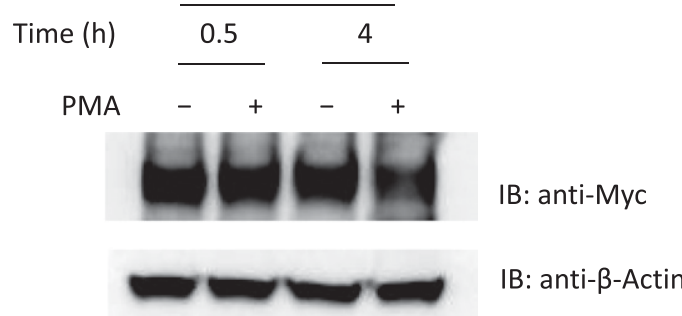

D

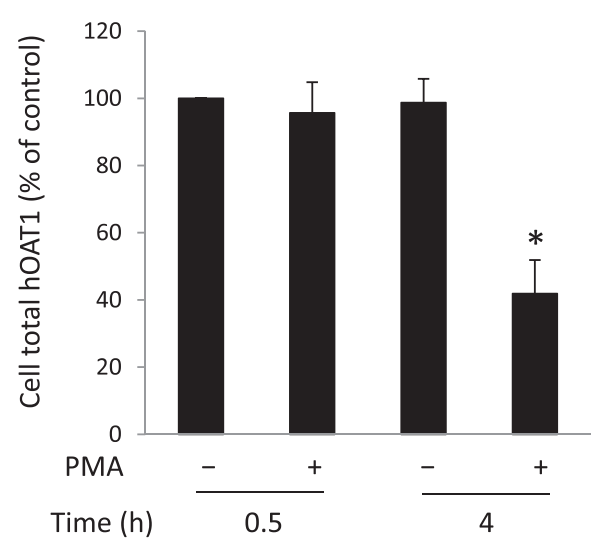

Fig. 1. Comparison of the short- and long-term effects of PKC on hOAT1 expression at the cell surface and in total cell lysates in COS-7 cells. (A) hOAT1 expression at the cell surface. (Top panel) hOAT1-expressing cells were treated with or without PKC activator PMA for 30 minutes and 4 hours, respectively. Cell surface biotinylation was then performed. Biotinylated/cell surface proteins were separated with streptavidin beads and analyzed by immunoblotting (IB) with an anti-myc antibody (myc was tagged to hOAT1 for immuno-detection). (Bottom panel) The same immunoblot from the top panel was reprobed by anti-E-cadherin antibody to determine the expression of the cell surface protein marker E-cadherin. (B) Densitometry plot of results from (A, top panel) as well as from other independent experiments. The values are mean \pm S.E. $(n=3)$. ${ }^{*} P<0.05$. (C) hOAT1 expression in total cell lysate. (Top panel) hOAT1-expressing cells were treated with or without PKC activator PMA for 30 minutes and 4 hours, respectively. Treated cells were lysed, and hOAT1 total expression was analyzed by IB with an anti-myc antibody. (Bottom panel) The same immunoblot from the top panel was reprobed by cell protein marker anti- $\beta$-actin antibody. (D) Densitometry plot of results from (C, top panel) as well as from other independent experiments. The values are mean \pm S.E. $(n=3) . * P<0.05$. 
A

hOAT1 expression at the cell surface
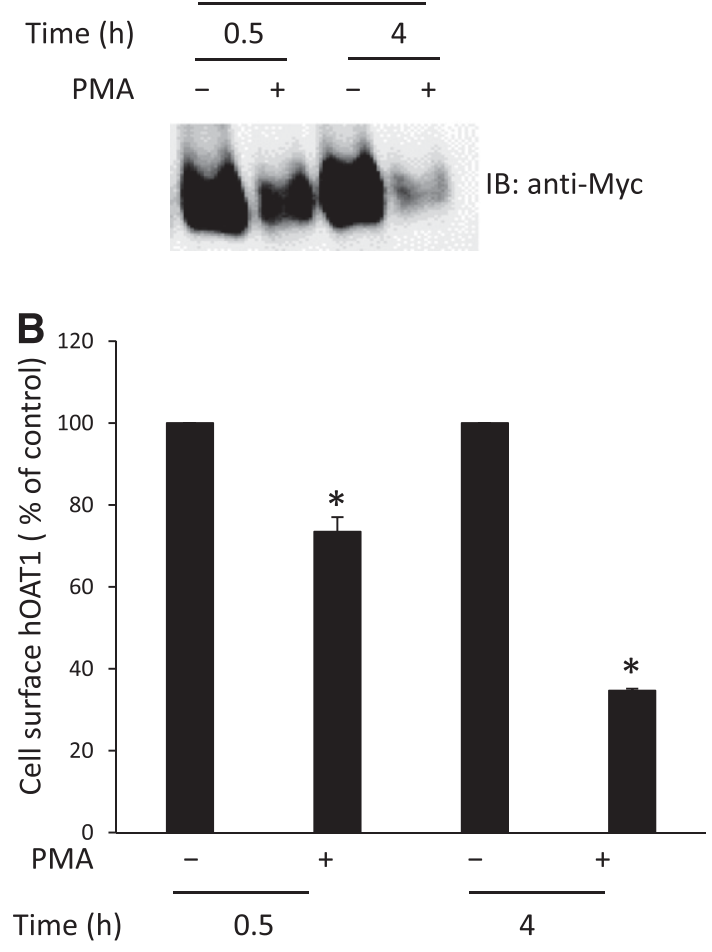

C hOAT1 expression in total cell lysates

Time (h)

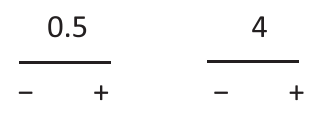

PMA

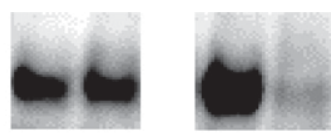

IB: anti-Myc

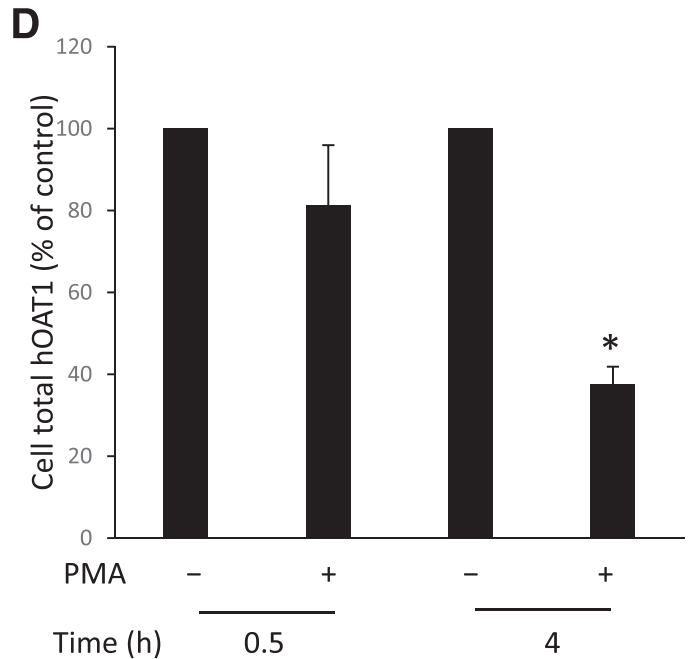

Fig. 2. Comparison of the short- and long-term effects of PKC on hOAT1 expression at the cell surface and in total cell lysates in HeLa cells. (A) hOAT1 expression at the cell surface. hOAT1-expressing cells were treated with or without PKC activator PMA for 30 minutes and 4 hours, respectively. Cell surface biotinylation was then performed. Biotinylated/cell surface proteins were separated with streptavidin beads and analyzed by immunoblotting (IB) with an anti-myc antibody (myc was tagged to hOAT1 for immunodetection). (B) Densitometry plot of results from (A) as well as from other independent experiments. The values are mean \pm S.E. $(n=3)$. $* P<0.05$. (C) hOAT1 expression in total cell lysate. hOAT1-expressing cells were treated with or without PKC activator PMA for 30 minutes and 4 hours, respectively. hOAT1 total expression was analyzed by IB with an antimyc antibody. (D) Densitometry plot of results from (C, top panel) as well as from other independent experiments. The values are mean \pm S.E. $(n=3)$. ${ }^{*} P<0.05$.

Degradation of Plasma Membrane hOAT1. hOAT1-expressing cells were labeled with NHS-SS-biotin as described previously. The labeled cells were then incubated with or without PMA at $37^{\circ} \mathrm{C}$. At designated time, cells were lysed and subjected to centrifugation at $16,000 \mathrm{~g}$ at $4^{\circ} \mathrm{C}$. The supernatant was mixed with streptavidin-agarose beads to isolate cell membrane proteins. The surface proteins were then separated on SDS-PAGE, followed by immunoblotting using an antimyc antibody to detect myc-tagged hOAT1.

Immunoprecipitation. Cells were lysed with lysis buffer $(10 \mathrm{mM}$ Tris/ $\mathrm{HCl}$, $\mathrm{pH} 7.5,10 \mathrm{mM} \mathrm{NaCl}, 0.5 \%$ Triton X-100, 2 mM EDTA, $10 \%$ glycerol, $1 \%$ of proteinase inhibitor cocktail, $20 \mathrm{mM} \mathrm{N}$-ethylmaleimide). Lysed cells were
A

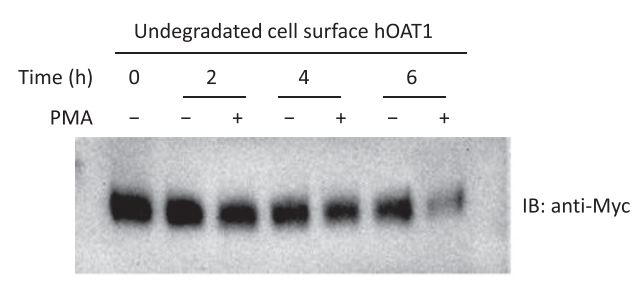

C

C

Undegradated cell surface Ecadherin

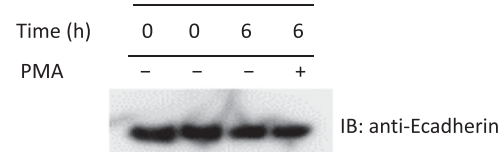

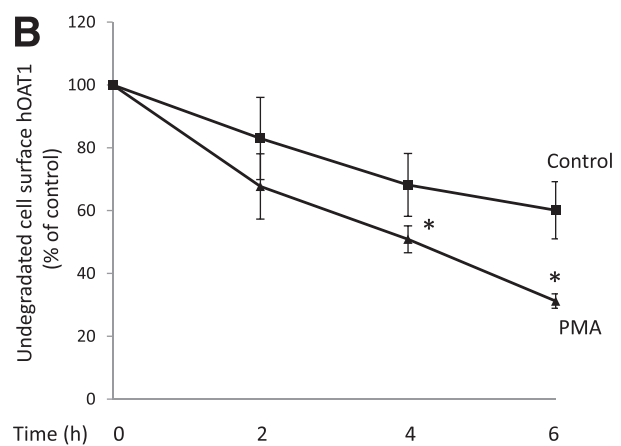

D

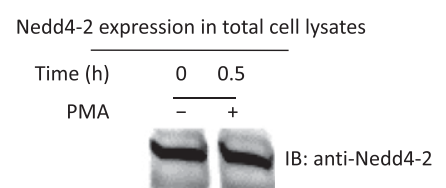

Fig. 3. Effect of $P K C$ activation on the degradation rate of cell surface hOAT1. (A) hOAT1-expressing cells were biotinylated with membrane impermeable biotinylation reagent sulfo-NHS-SS-biotin. Labeled cells were then treated with or without PKC activator PMA at $37^{\circ} \mathrm{C}$ for 2,4 , and 6 hours, respectively. Treated cells were lysed and cell surface proteins were isolated using streptavidin-agarose beads, followed by immunoblotting (IB) with anti-myc antibody. (B) Densitometry plot of results from (A) as well as from other independent experiments. The values are mean \pm S.E. $(n=3)$. $* P<0.05$ (between PMA-treated and control at the same time point). (C) hOAT1-expressing cells were biotinylated with membrane impermeable biotinylation reagent sulfo-NHS-SSbiotin. Labeled cells were then treated with or without PKC activator PMA at $37^{\circ} \mathrm{C}$ for 6 hours. Treated cells were lysed and cell surface proteins were isolated using streptavidinagarose beads, followed by IB with anti-Ecadherin antibody. (D) hOAT1-expressing cells were treated with or without PKC activator PMA at $37^{\circ} \mathrm{C}$ for 30 minutes. Treated cells were lysed, followed by IB with anti-Nedd4-2 antibody. 
A
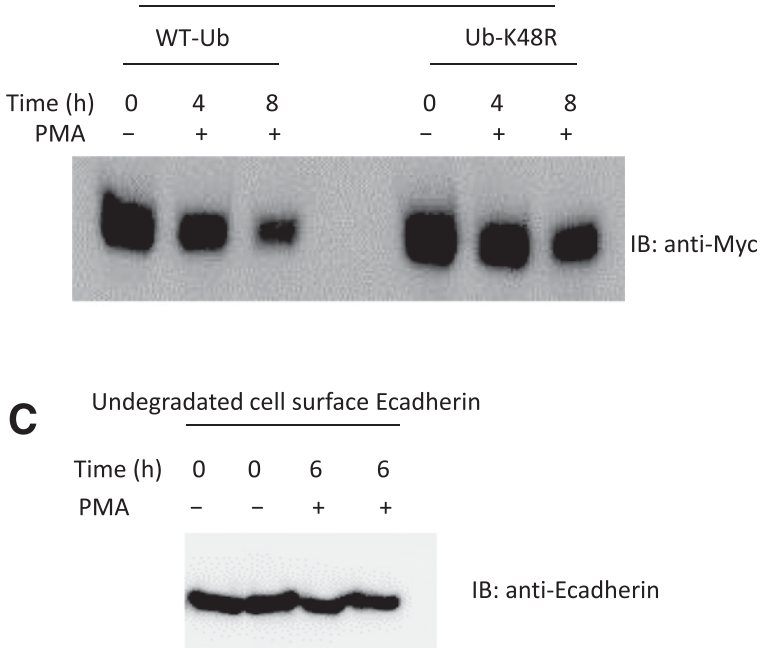

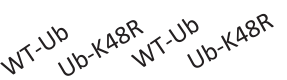

B

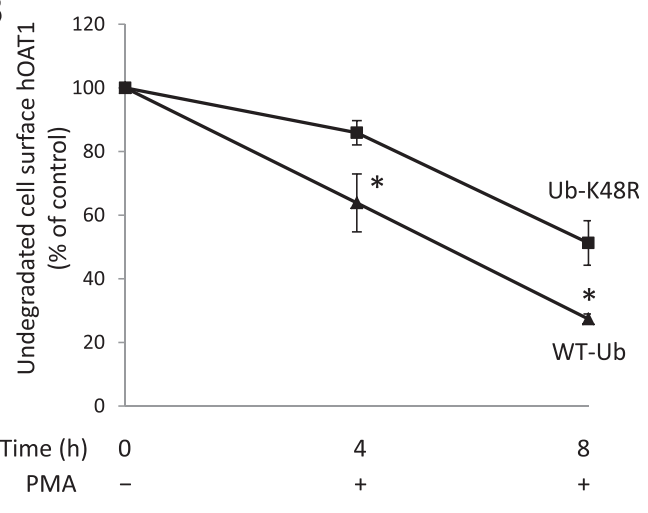

Fig. 4. Effect of ubiquitin mutant Ub-K48R on the degradation rate of cell surface hOAT1. (A) hOAT1-expressing cells were transfected with wild-type ubiquitin (WT-Ub) or ubiquitin mutant (Ub-K48R). Transfected cells were biotinylated with membrane impermeable biotinylation reagent sulfo-NHS-SS-biotin. Labeled cells were then treated with or without PKC activator PMA at $37^{\circ} \mathrm{C}$ for 4 or 8 hours, respectively. Cells were lysed afterward and cell surface proteins were isolated using streptavidin-agarose beads, followed by immunoblotting (IB) with anti-myc antibody. (B) Densitometry plot of results from (A) as well as from other independent experiments. The values are mean \pm S.E. $(n=3)$. $* P<$ 0.05 (between WT-Ub- and Ub-K48R-transfected cells at the same time point). (C) hOAT1-expressing cells were transfected with WT-Ub or Ub-K48R. Transfected cells were biotinylated with membrane impermeable biotinylation reagent sulfo-NHS-SS-biotin. Labeled cells were then treated with or without PKC activator PMA at $37^{\circ} \mathrm{C}$ for 6 hours. Cells were lysed afterward and cell surface proteins were isolated using streptavidin-agarose beads, followed by IB with anti-E-cadherin antibody.

precleared at $4^{\circ} \mathrm{C}$ with protein $\mathrm{G}$-agarose beads to minimize nonspecific binding. The precleared lysate was then mixed overnight with antibodybound protein G-agarose beads. Proteins bound to the protein G-agarose beads were eluted with urea buffer, followed by immunoblotting with appropriate antibodies.
Electrophoresis and Immunoblotting. Protein samples were separated on 7.5\% SDS-PAGE minigel, and electroblotted onto polyvinylidene difluoride membranes, followed by incubation overnight with appropriate primary antibodies, and subsequently by incubation with horseradish peroxidase-conjugated secondary antibodies. The SuperSignal West Dura Extended Duration Substrate
A

Undegradated cell surface hOAT1

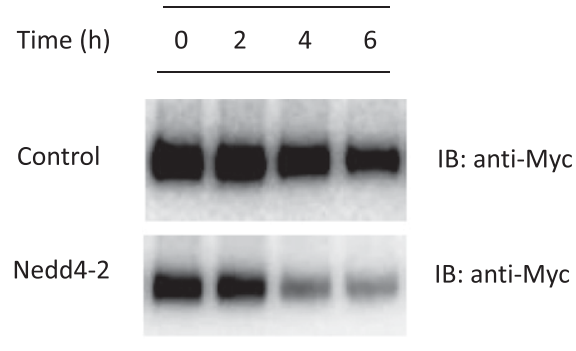

C Undegradated cell surface Ecadherin
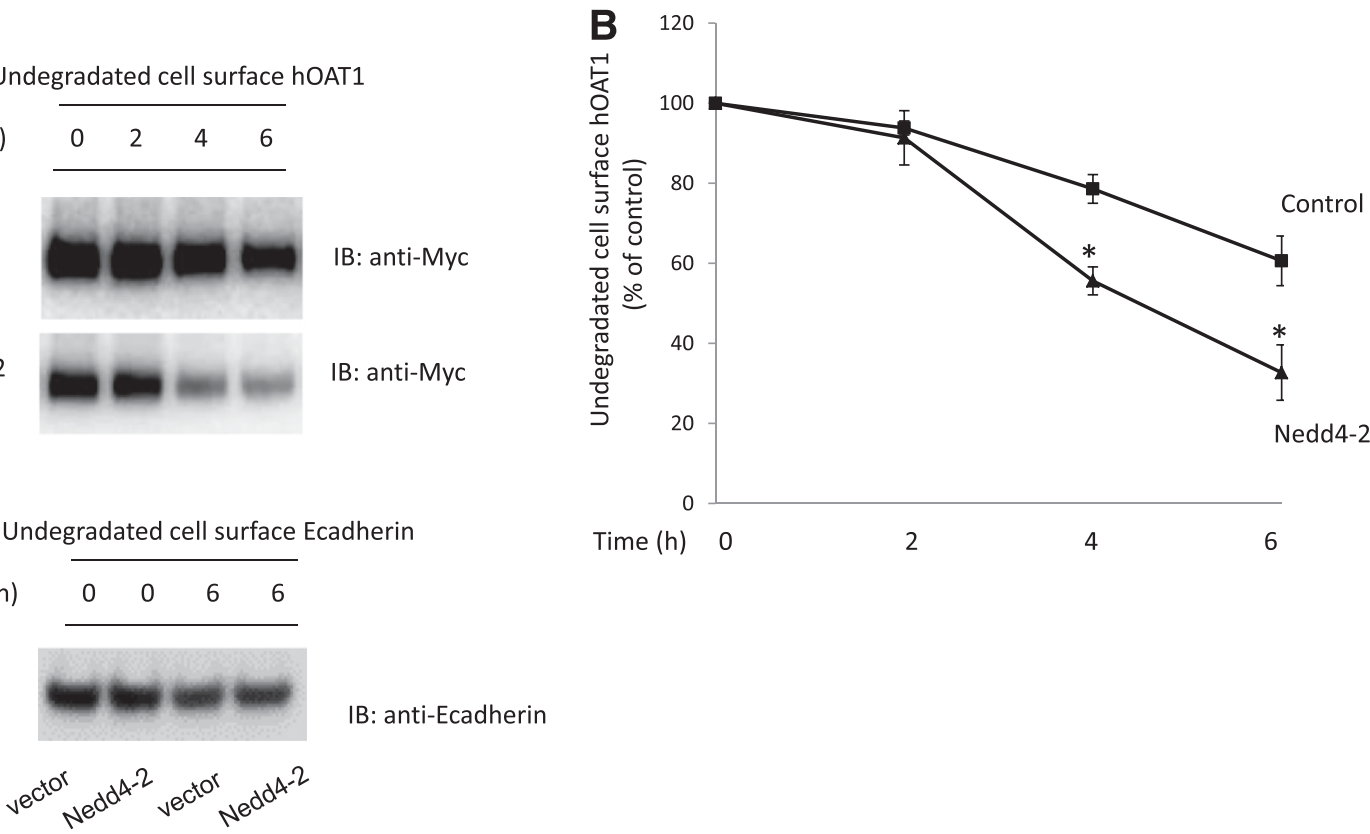

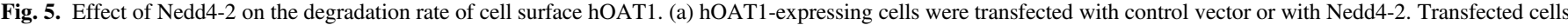

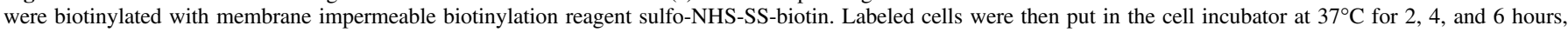

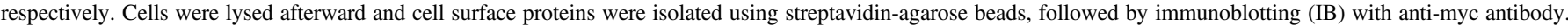

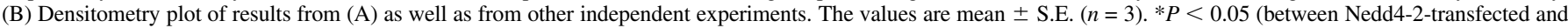

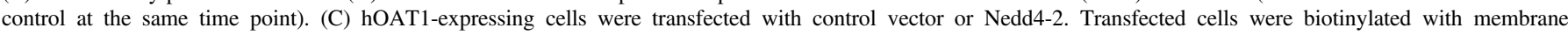

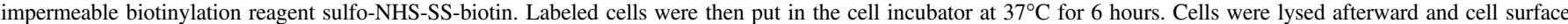
proteins were isolated using streptavidin-agarose beads, followed by IB with anti-E-cadherin antibody. 
kit (Pierce) was used to detect the signals, followed by quantification using scanning densitometry with the FluorChem 8000 imaging system (Alpha Innotech Corp., San Leandro, CA).

Data Analysis. We performed each experimental test at least three times, and multiple experiments were used to carry out the statistical analysis using Student's paired $t$ tests. An asterisk indicates a value of $P<0.05$.

\section{Results}

Effect of Long-Term PKC Activation on hOAT1 Expression. We previously demonstrated (Zhang et al., 2008) that short-term treatment ( $<30$ minutes) of cells with PKC activator PMA leads to inhibition of hOAT1-mediated transport by accelerating hOAT1 internalization from the plasma membrane to intracellular endosomes. Consequently, the amount of hOAT1 at the plasma membrane is reduced, and hOAT1 transport activity is decreased. In the current investigation, we examined the effect of long-term PKC activation on hOAT1 expression. hOAT1expressing cells were treated with PKC activator PMA for 30 minutes and 4 hours, respectively, and hOAT1 expression at the plasma membrane and in total cell lysates was then compared. Our results showed that (Fig. 1A, top panel; Fig. 1B) both short-term treatment (30 minutes) and long-term treatment (4 hours) with PMA led to a decrease in hOAT1 expression at the plasma membrane. Furthermore, such a change in hOAT1 cell surface expression was not a result of the overall perturbation of plasma membrane proteins because there was no significant change in the expression of the cell surface protein marker E-cadherin (Fig. 1A, bottom panel). In contrast, short-term PMA treatment had no effect on the total expression of hOAT1 (Fig. 1C, top panel; Fig. 1D), whereas long-term treatment (4 hours) with PMA resulted in significant reduction of hOAT1 expression in total cell lysate (Fig. 1C, top panel; Fig. 1D). Again, such a change in hOAT1 total expression was not a result of the general perturbation of cellular proteins because there was no change in the expression of cellular protein marker $\beta$-actin (Fig. $1 \mathrm{C}$, bottom panel). The decrease in the total expression of hOAT1 after long-term PMA treatment suggests that hOAT1 stability was affected. Comparable results were obtained in HeLa cells (Fig. 2), suggesting that PKC regulation of hOAT1 is not cell type specific.

Effect of Long-Term PKC Activation on hOAT1 Stability. The stability of cell surface hOAT1 was subsequently assessed using a biotinylation approach. hOAT1-expressing cells were labeled with membrane-impermeable biotinylation reagent sulfo-NHS-SS-biotin. Labeled cells were then incubated with or without PKC activator PMA at $37^{\circ} \mathrm{C}$ for 2,4 , and 6 hours, respectively. After the treatment, cells were lysed and cell surface proteins were purified using
A
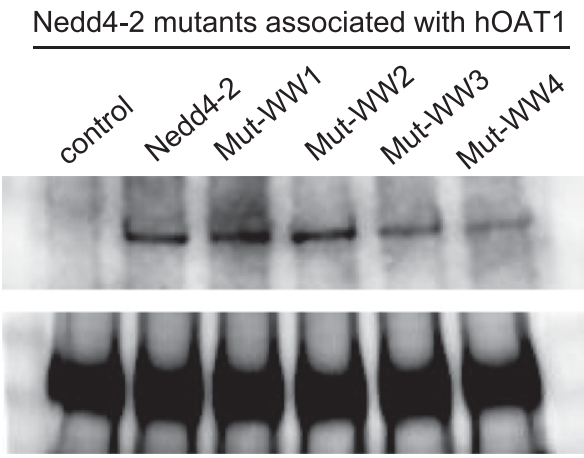

IP: anti-Myc

IB: anti-Myc

IP: anti-Myc
C Nedd4-2 mutant associated with hOAT1

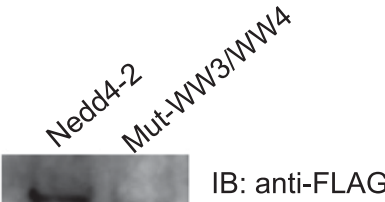

IP: anti-Myc

IB: anti-Myc

IP: anti-Myc
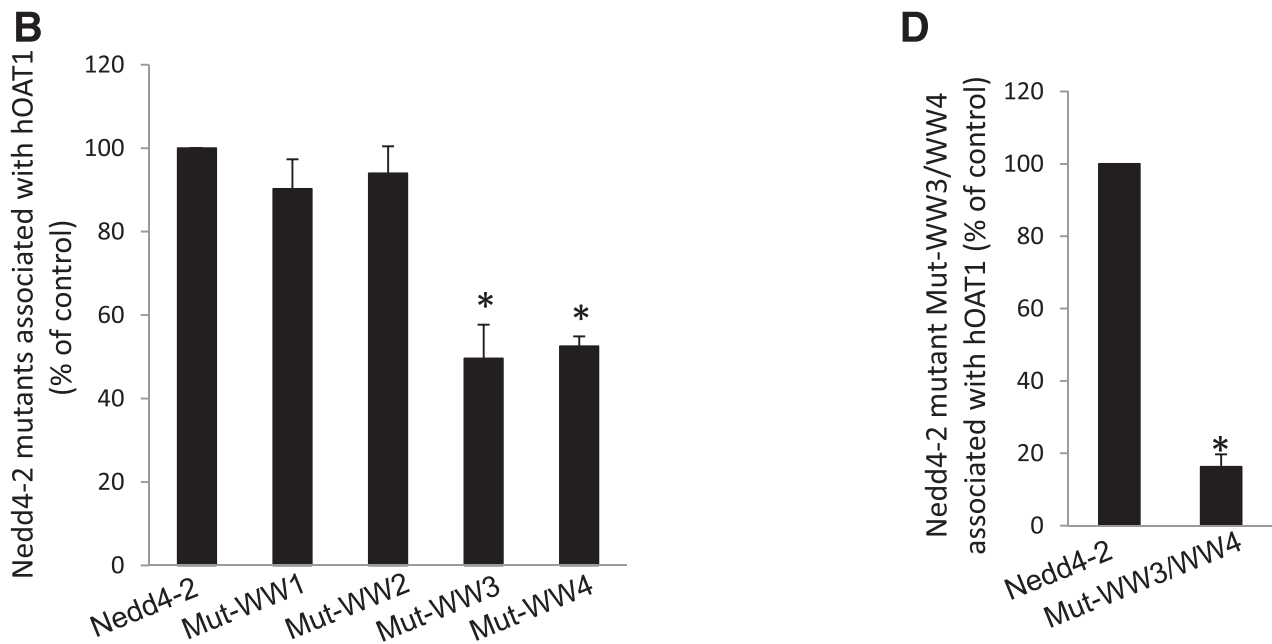

Fig. 6. Interaction between Nedd4-2 WW domain mutants and hOAT1. (A, top panel) Two amino acid residues in each of the four WW domains of Nedd4-2 were mutated (Mut-WW1: V91W/H93G; Mut-WW2: V283W/H285G; Mut-WW3: I395W/H397G; and Mut-WW4: I446W/H448G). Mutant-transfected cells were lysed, and hOAT1 was then immunoprecipitated (IP) with anti-myc antibody, followed by immunoblotting (IB) with anti-FLAG antibody. Epitope FLAG was tagged to wild-type Nedd4-2 and its mutants for immunodetection of exogenously expressed Nedd4-2 and its mutants. (Bottom panel) The same immunoblot from the top panel was reprobed by anti-myc antibody to determine the amount of hOAT1 IP. (B) Densitometry plot of results from (A, top panel) as well as from other independent experiments. The values are mean \pm S.E. $(n=3)$. $* P<0.05$. (C, top panel) WW domain 3 and WW domain 4 were simultaneously mutated (Mut-WW3/WW4). Wild-type Nedd4-2- and mutant MutWW3/WW4-transfected cells were lysed, and hOAT1 was then IP with anti-myc antibody, followed by IB with anti-FLAG antibody. (Bottom panel) The same immunoblot from the top panel was reprobed by anti-myc antibody to determine the amount of hOAT1 IP. (D) Densitometry plot of results from (C, top panel) as well as from other independent experiments. The values are mean \pm S.E. $(n=3) . * P<0.05$. 
streptavidin-agarose beads, followed by immunoblotting using anti-myc antibody (epitope myc was tagged to hOAT1 for immunodetection). Our results (Fig. 3, A and B) showed that the rate of hOAT1 degradation increased significantly after 2-hour PMA treatment compared with that of control. After 6-hour treatment with PMA, $~ 70 \%$ of hOAT1 was degraded compared with only $\sim 30 \%$ of hOAT 1 degradation in control samples. These results indicate that long-term PKC activation significantly decreases hOAT1 stability. Under the same condition, the expression of cell surface protein marker E-cadherin was not affected by PMA treatment (Fig. 3C). Our previously published work demonstrated that Nedd4-2, a ubiquitin ligase, mediates the PKC regulation of hOAT1 expression and activity (Xu et al., 2016a). We show here that the expression of Nedd4-2 is not affected by PMA treatment (Fig. 3D).

The Role of K48-Linked Polyubiquitin Chains in PKCRegulated hOAT1 Degradation. Our published work (Zhang et al., 2008) revealed that short-term PKC activation accelerates hOAT1 internalization from the cell surface by promoting the conjugation of the K48-linked polyubiquitin chain to the transporter. To explore the role of K48-linked polyubiquitin chains in long-term PKC regulation of hOAT1 stability, we transfected cells with wild-type ubiquitin or ubiquitin mutant Ub-K48R. Ub-K48R has the lysine $(\mathrm{K})$ at position 48 mutated to arginine $(\mathrm{R})$ and therefore prevents the formation of K48linked polyubiquitin chains. Transfected cells were then labeled with membrane impermeable biotinylation reagent sulfo-NHS-SS-biotin, followed by treatment with or without PKC activator PMA for 4 and 8 hours, respectively. The degradation rates of cell surface hOAT1 in these cells were then determined as described previously. As shown in Fig. 4, A and B, the degradation of hOAT1 was much slower in mutant Ub-K48R-transfected cells than that in wild-type ubiquitin-transfected cells. These results indicate that conjugation of the K48-linked polyubiquitin chain to hOAT1 plays a significant role in $\mathrm{PKC}$ regulation of hOAT1 stability. Under the same condition, the expression of cell surface protein marker E-cadherin was not affected by the expression of wild-type ubiquitin and Ub-K48R (Fig. 4C).

The Effect of Nedd4-2 on hOAT1 Stability. We previously established that PKC-stimulated ubiquitination of hOAT1 is catalyzed by ubiquitin ligase Nedd4-2 (Xu et al., 2016b). In this experiment, we examined the effect of Nedd4-2 on hOAT1 stability. hOAT1-expressing cells were transfected with or without Nedd4-2. The degradation rates of plasma membrane hOAT1 in these cells were then determined using the biotinylation approach as described previously. As shown in Fig. 5, A and B, the degradation of hOAT1 was much faster in Nedd4-2transfected cells than that in control cells, suggesting that Nedd4-2mediated ubiquitination plays an important role in hOAT1 stability. Under the same condition, the expression of cell surface protein marker E-cadherin was not affected by the transfection of Nedd4-2 (Fig. 5C).

Interaction of Nedd4-2 with hOAT1. Nedd4-2 interacts with its target proteins, either directly or indirectly, through its four WW domains (WW1-4). We mutated two amino acids in each domain separately (Mut-WW1: V91W/H93G; Mut-WW2: V283W/H285G; Mut-WW3: I395W/H397G; and Mut-WW4: I446W/H448G). It was previously proven that the mutation of these amino acids inactivates the WW domains, and thus interferes with the binding of Nedd4-2 to its substrates. The cDNAs of Nedd4-2 mutants were transfected into the cells. hOAT1 was then pulled down by anti-myc antibody (myc was tagged to hOAT1), and subjected to immunoblotting with anti-FLAG antibody. Epitope FLAG was tagged to wild-type Nedd4-2 and its mutants so that the exogenously expressed Nedd4-2 and its mutants could be immunodetected. As shown in Fig. 6, although wild-type Nedd4-2 and its mutants were all efficiently and equally expressed in hOAT1-expressing cells (data not shown), the amounts of Mut-WW3 and Mut-WW4 detected in hOAT1 immunoprecipitates were much less in comparison with that of wild-type Nedd4-2, Mut-WW1, and MutWW2 (Fig. 6A, top panel; Fig. 6B), indicating that less amounts of MutWW3 and Mut-WW4 were associated with hOAT1. The association of Nedd4-2 with hOAT1 was further reduced when its WW3 and WW4 domains were mutated simultaneously (Mut-WW3/WW4) (Fig. 6C, top panel; Fig. 6D). The different amounts of Nedd4-2 mutants detected in hOAT1 immunoprecipitates did not result from different amounts of hOAT1 immunoprecipitated because there were similar amounts of hOAT1 immunoprecipitated in all samples under these conditions (Fig. 6A, bottom panel; Fig. 6C, bottom panel). These results suggest that WW3 and WW4 domains are critical for the binding of Nedd4-2 to hOAT1.

Effect of Mutations of Nedd4-2 WW Domains on hOAT1 Ubiquitination. The data shown previously (Fig. 6) demonstrated that mutations at the WW3 and WW4 domains of Nedd4-2 weakened the binding of Nedd4-2 to hOAT1. To test whether such mutations affect hOAT1 ubiquitination, we transfected cells with cDNAs for wild-type Nedd4-2 or Nedd4-2 WW domain mutant Mut-WW3/WW4. hOAT1 was then pulled down by anti-myc antibody, followed by immunoblotting with anti-ubiquitin antibody. Our results showed that (Fig. 7A, top panel; Fig. 7B) hOAT1 ubiquitination was significantly decreased in Mut-WW3/WW4-transfected cells in comparison with that in wild-type Nedd4-2-transfected cells. Furthermore, the different amounts of ubiquitinated hOAT1 were not due to the different amounts of hOAT1 immunoprecipitated because there were similar amounts of hOAT1 immunoprecipitated in both samples under the same conditions (Fig. 7A, bottom panel). These data suggest that the decreased binding affinity of Mut-WW3/WW4 for hOAT1 results in decreased hOAT1 ubiquitination.

The Effect of Mutations of WW Domains in Nedd4-2 on the Cell Surface Expression of hOAT1. We next assessed the effect of wildtype Nedd4-2 and its mutant Mut-WW3/WW4 on the cell surface expression of hOAT1. Our results (Fig. 8A, top panel; Fig. 8B) showed that hOAT1 expression was $\sim 30 \%$ lower in wild-type Nedd4-2transfected cells compared with that in control cells. Nedd4-2 mutant Mut-WW3/WW4 significantly reversed the effect of wild-type Nedd4-2
A

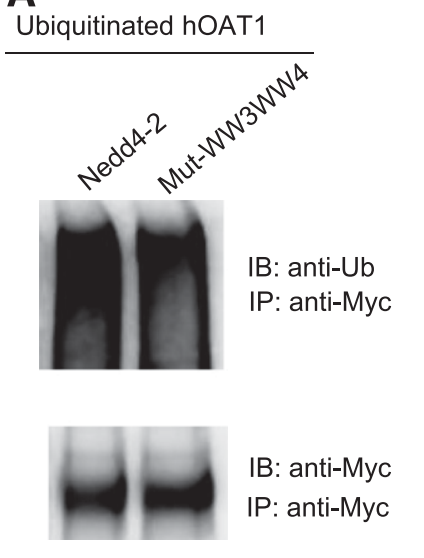

B

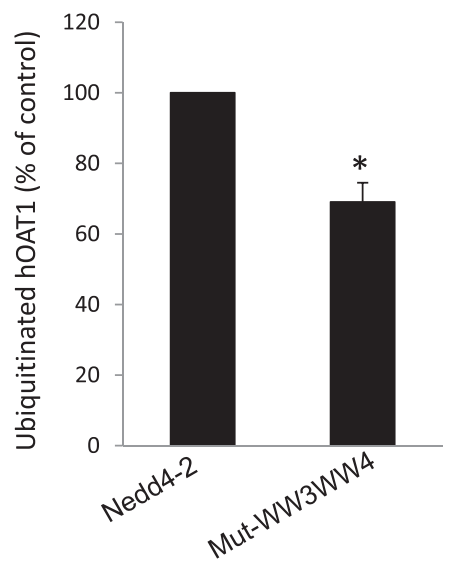

Fig. 7. Effect of Nedd4-2 WW domain mutant Mut-WW3/WW4 on hOAT1 ubiquitination. (A, top panel) hOAT1-expressing COS-7 cells were transfected with cDNAs for wild-type Nedd4-2 or for Nedd4-2 WW domain mutant (MutWW3/WW4). Transfected cells were then lysed, and hOAT1 was immunoprecipitated (IP) with anti-myc antibody, followed by immunoblotting (IB) with anti-ubiquitin antibody (anti-Ub). (Bottom panel) The blot from the top panel was reprobed with antimyc antibody to determine the amount of hOAT1 IP. (B) Densitometry plot of results from (A, top panel) as well as from other independent experiments. The values are mean \pm S.E. $(n=3)$. $* P<0.05$. 
A

Cell surface expression of hOAT1

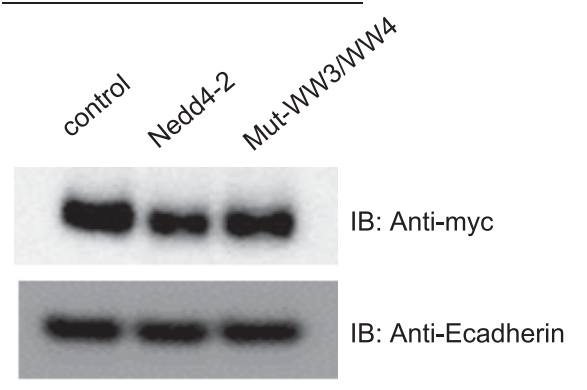

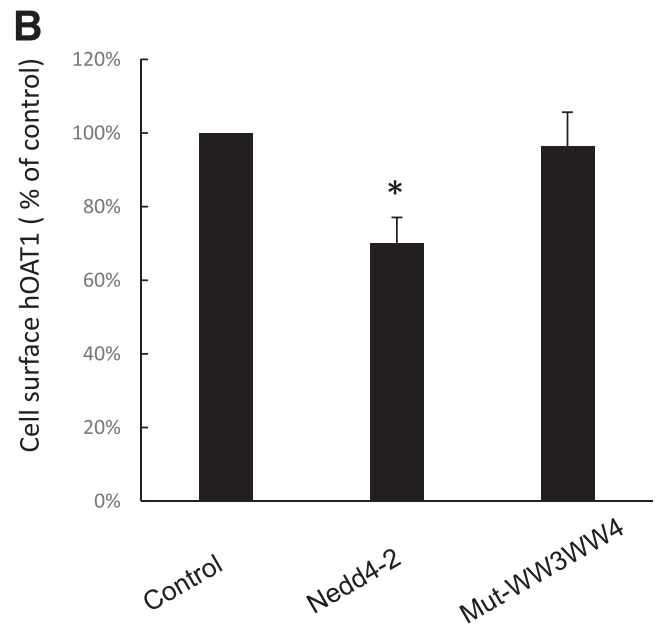

Fig. 8. Effect of Nedd4-2 WW domain mutant Mut-WW3/WW4 on hOAT1 cell surface expression. (A, top panel) hOAT1-expressing COS-7 cells were transfected with cDNAs for wild-type Nedd4-2 or for Nedd4-2 WW domain mutant (Mut-WW3/WW4). Cell surface biotinylation was then performed. Biotinylated/cell surface proteins were separated with streptavidin beads and analyzed by immunoblotting (IB) with an anti-myc antibody. (Bottom panel) The same immunoblot from the top panel was reprobed by anti-E-cadherin antibody to determine the expression of the cell surface protein marker E-cadherin. (B) Densitometry plot of results from (A, top panel) as well as from other independent experiments. The values are mean \pm S.E. $(n=$ 3). $* P<0.05$ on hOAT1 expression. The expression of cell surface protein marker E-cadherin was not affected by the transfection of wild-type Nedd4-2 or its mutant Mut-WW3/WW4 (Fig. 8A, bottom panel).

The Effect of Mutations of WW Domains in Nedd4-2 on hOAT1 Transport Activity. To explore whether WW domain mutations of Nedd4-2 affect hOAT1 function, we measured hOAT1-mediated transport of ${ }^{3} \mathrm{H}$-labeled $p$-aminohippuric acid in cells transfected with cDNAs for wild-type Nedd4-2 or its mutant Mut-WW3/WW4. We showed that (Fig. 9) overexpression of wild-type Nedd4-2 inhibited hOAT1mediated transport $\sim 40 \%$, whereas such a level of inhibition was partially reversed in cells transfected with Mut-WW3/WW4.

\section{Discussion}

hOAT1 plays a pivotal role in drug efficacy and toxicity. The transport activity of hOAT1 is critically dependent on its expression level at the plasma membrane. We previously established that hOAT1 at the plasma membrane is dynamic, constitutively internalizing from and recycling back to the plasma membrane. Short-term (30 minutes) PKC activation inhibits hOAT1 transport activity by promoting the attachment of a lysine 48-linked polyubiquitin chain to hOAT1, a step catalyzed by ubiquitin ligase Nedd4-2. The polyubiquitin chain attached to hOAT1 can be recognized by endocytosis machinery and triggers an accelerated endocytosis of the transporter from the plasma membrane. Consequently, the amount of hOAT1 at the plasma membrane is reduced and hOAT1 transport activity is decreased. In the present study, we investigated the long-term effect of PKC on hOAT1.

To carry out our investigation, we chose monkey kidney COS-7 cells, a model cell system that has been widely used for mechanistic studies of many kidney transport processes. Our studies in these cells will pave the path for future exploration in assessing whether similar mechanisms are working in vivo.

From our investigation, we obtained several pieces of important information. First, there was a sharp contrast between the short- and long-term effects of PKC on hOAT1: short-term PKC activation promoted hOAT1 to traffic from the plasma membrane to intracellular endosomes without influencing the total expression of the transporter. However, long-term activation of PKC led hOAT1 to degrade. Second, PKC-induced hOAT1 degradation was partially blocked by ubiquitin mutant Ub-K48R. Ub-K48R is defective in forming the K48-linked polyubiquitin chain. Third, the ubiquitin ligase Nedd4-2 also promoted hOAT1 degradation. These results suggest that Nedd4-2-catalyzed conjugation of the K48-linked polyubiquitin chain to hOAT1 not only plays an important role in short-term PKC regulation of hOAT1 trafficking but also is critical in the long-term PKC effect on hOAT1 stability. These observations provide important insights into the molecular basis underlying the defective drug transport in bilateral ureteral obstruction. Clinically, bilateral ureteral obstruction causes acute renal failure (Klahr, 1998; Klahr and Morrissey, 2002). In the rat model of this disease, elimination of drugs was impaired partly as a result of decreased OAT1 expression (Villar et al., 2005). In bilateral ureteral obstruction the level of angiotensin II is elevated (Klahr, 1998; Klahr and Morrissey, 2002), and it was shown that angiotensin II is a physiologic PKC activator (Duan et al., 2010). Therefore, angiotensin II may degrade OAT1 through Nedd4-2-mediated PKC regulation of OAT1 ubiquitination.

Our present studies also indicate that the effect of Nedd4-2 on hOAT1 partly arises from a direct association between the WW-3/WW-4 domains of Nedd4-2 and the transporter. The WW domains are well known to bind proline-rich sequences in the target proteins (Espanel and Sudol, 1999; Kamynina et al., 2001; Fotia et al., 2003; Vina-Vilaseca

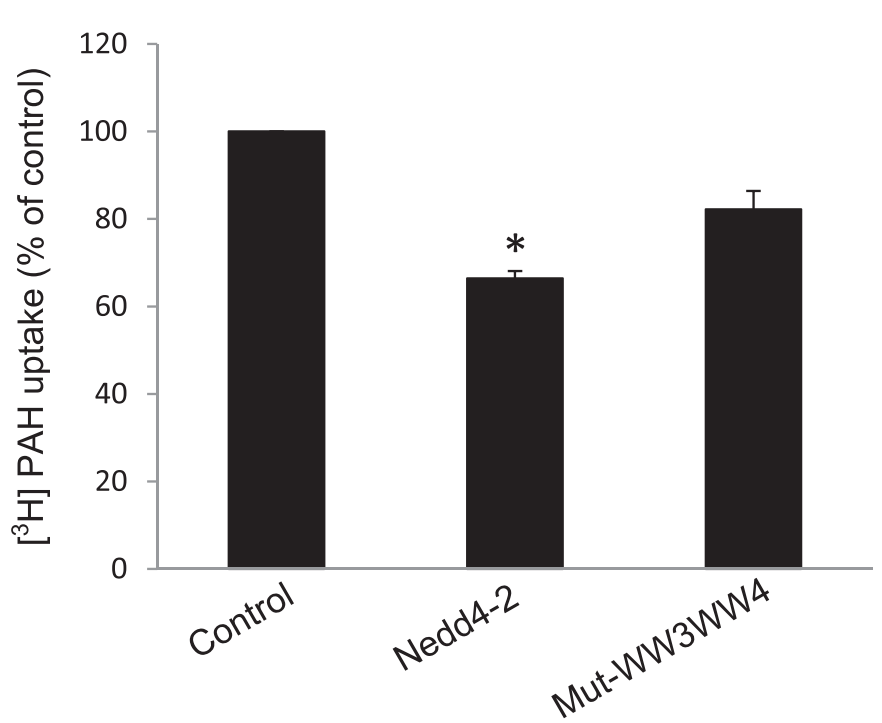

Fig. 9. Effect of Nedd4-2 WW domain mutant on hOAT1 transport activity. hOAT1-expressing cells were transfected with cDNAs for wild-type Nedd4-2 or for Nedd4-2 WW domain mutant Mut-WW3/WW4. Transfected cells were then measured for 3-minute uptake of $\left[{ }^{3} \mathrm{H}\right] p$-aminohippuric acid (PAH) $(20 \mu \mathrm{M})$ at room temperature. Uptake activity was expressed as a percentage of the uptake measured in control cells. The data represent uptake into hOAT1-transfected cells minus uptake into mock cells (parental cells). Values are mean \pm S.E. $(n=3)$. ${ }^{*} P<0.05$. 


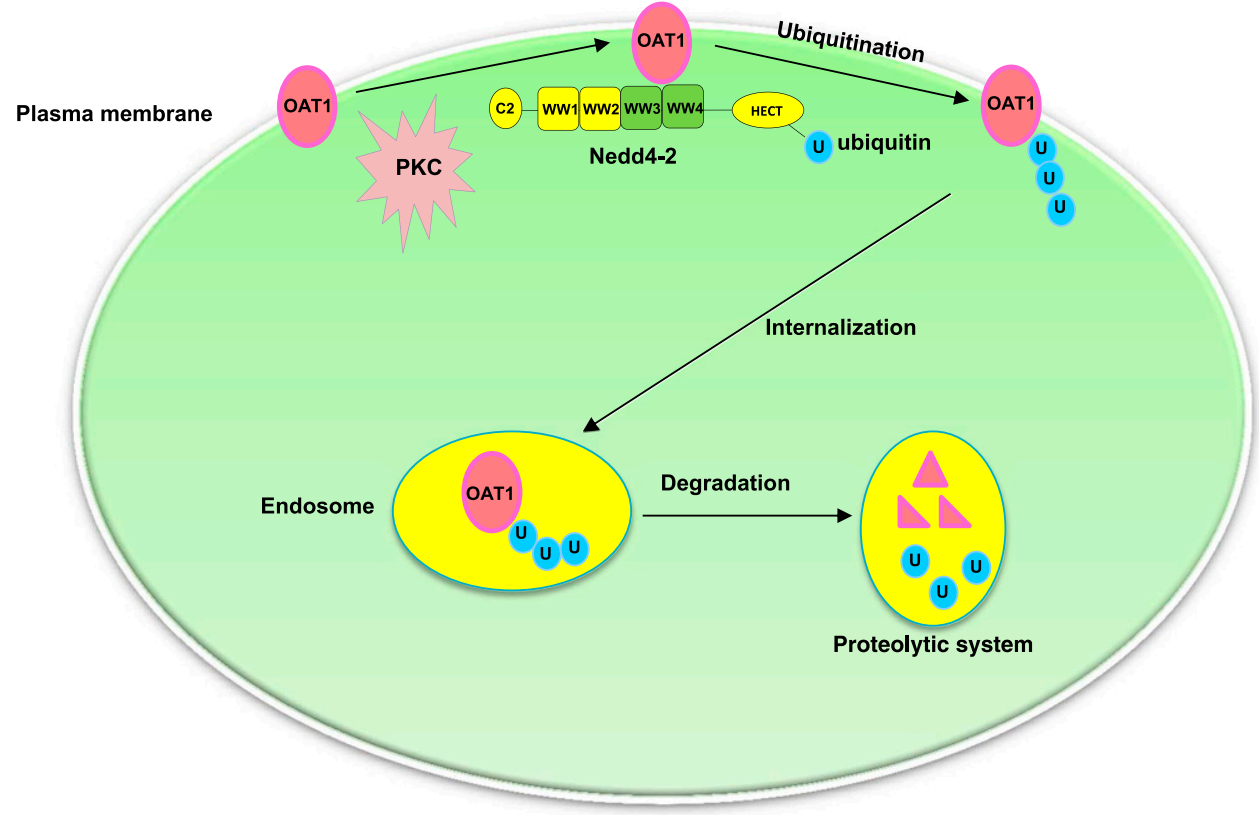

Fig. 10. $\mathrm{PKC} / \mathrm{Nedd} 4-2$ signaling pathway regulates the activity of drug transporter hOAT1.

and Sorkin, 2010). There are four WW domains in Nedd4-2. These domains are known to have different selectivity and affinity to its target proteins. For instance, Nedd4-2 plays critical roles in the function of the epithelial sodium channel, and its inhibition of epithelial sodium channel activity depends on WW domains 3 and 4, and to a lesser extent on WW domain 2 (Harvey et al., 1999; Farr et al., 2000). Yet, WW domains 1 and 2 of Nedd4-2 are important for ubiquitinating calcium channel TRPV6 (Zhang et al., 2010). Our present study also uncovered that the binding affinity of the WW domains of Nedd4-2 to hOAT1 was substantially selective. WW domains 3 and 4, but not WW domains 1 and 2, were essential for the association of Nedd4-2 to hOAT1. When WW domains 3 and 4 in Nedd4-2 were inactivated individually or in combination through site-directed mutagenesis, the binding affinity of these Need4-2 mutants (Mut-WW3, Mut-WW4, and Mut-WW3/WW4) for hOAT1 was significantly weakened (Fig. 6). We noticed that the effect of Mut-WW3/WW4 on hOAT1 ubiquitination, cell surface expression, and transport activity was not as dramatic as the effect on their binding to hOAT1 (Figs. 7-9). The possible explanation is that in the study examining the binding of Mut-WW3/WW4 to hOAT1 only the FLAG-tagged exogenously expressed Nedd4-2 mutant was probed. In the study examining the effect of Mut-WW3/WW4 on hOAT1 ubiquitination and transport activity we were looking at the combinational effect of Mut-WW3/WW4 and the endogenously expressed and fully functional Nedd4-2.

Finally, it should be noted that hOAT1 was shown in our study to interact with Nedd4-2 despite the lack of conventional WW domainbinding (L/P)PXY motifs. This indicates that the interaction occurs through noncanonical sequences in hOAT1. This is consistent with findings from other laboratories that Nedd4-2 is physically associated with the glutamate transporter GLT-1 (García-Tardón et al., 2012) and mammalian dopamine transporter DAT (Sorkina et al., 2006), none of which possesses the conventional WW domainbinding motifs.

In conclusion, we provided the first demonstration that Nedd4-2catalyzed conjugation of a lysine 48-linked polyubiquitin chain to hOAT1 plays a significant role in the long-term PKC regulation of hOAT1 stability, and that WW domains 3 and 4 of Nedd4-2 are critical in its association with and regulation of the transporter (Fig. 10). Our study offers important insights into understanding the molecular and cellular foundation of hOAT1 regulation in vivo.

\section{Acknowledgments}

We thank Zhongyang Shi for assistance with the experiments.

\section{Authorship Contributions}

Participated in research design: Xu, J. Zhang, You.

Conducted experiments: Xu, J. Zhang, Q. Zhang, Fan, Liu.

Performed data analysis: Xu, J. Zhang, You.

Wrote or contributed to the writing of the manuscript: Xu, You.

\section{References}

Ahn SY and Nigam SK (2009) Toward a systems level understanding of organic anion and other multispecific drug transporters: a remote sensing and signaling hypothesis. Mol Pharmacol 76:481-490. Duan P, Li S, and You G (2010) Angiotensin II inhibits activity of human organic anion transporter 3 through activation of protein kinase $\mathrm{C} \alpha$ : accelerating endocytosis of the transporter. Eur $J$ Pharmacol 627:49-55.

Espanel X and Sudol M (1999) A single point mutation in a group I WW domain shifts its specificity to that of group II WW domains. J Biol Chem 274:17284-17289.

Farr TJ, Coddington-Lawson SJ, Snyder PM, and McDonald FJ (2000) Human Nedd4 interacts with the human epithelial $\mathrm{Na}^{+}$channel: WW3 but not WW1 binds to $\mathrm{Na}^{+}$-channel subunits. Biochem J 345:503-509.

Fotia AB, Dinudom A, Shearwin KE, Koch JP, Korbmacher C, Cook DI, and Kumar S (2003) The role of individual Nedd4-2 (KIAA0439) WW domains in binding and regulating epithelia sodium channels. FASEB $J$ 17:70-72.

García-Tardón N, González-González IM, Martínez-Villarreal J, Fernández-Sánchez E, Giménez C, and Zafra F (2012) Protein kinase C (PKC)-promoted endocytosis of glutamate transporter GLT-1 requires ubiquitin ligase Nedd4-2-dependent ubiquitination but not phosphorylation. $J$ Biol Chem 287:19177-19187.

Harvey KF, Dinudom A, Komwatana P, Jolliffe CN, Day ML, Parasivam G, Cook DI, and Kumar S (1999) All three WW domains of murine Nedd4 are involved in the regulation of epithelial sodium channels by intracellular $\mathrm{Na}^{+}$. J Biol Chem 274:12525-12530.

Kamynina E, Tauxe C, and Staub O (2001) Distinct characteristics of two human Nedd4 protein with respect to epithelial $\mathrm{Na}^{+}$channel regulation. Am J Physiol Renal Physiol 281:F469-F477. Klahr S (1998) Obstructive nephropathy. Kidney Int 54:286-300.

Klahr S and Morrissey J (2002) Obstructive nephropathy and renal fibrosis. Am J Physiol Renal Physiol 283:F861-F875.

Miranda M and Sorkin A (2007) Regulation of receptors and transporters by ubiquitination: new insights into surprisingly similar mechanisms. Mol Interv 7:157-167.

Pelis RM and Wright SH (2011) Renal transport of organic anions and cations. Compr Physiol 1: 1795-1835.

Snyder PM, Steines JC, and Olson DR (2004) Relative contribution of Nedd4 and Nedd4-2 to ENaC regulation in epithelia determined by RNA interference. $J$ Biol Chem 279:5042-5046.

Sorkina T, Miranda M, Dionne KR, Hoover BR, Zahniser NR, and Sorkin A (2006) RNA interference screen reveals an essential role of Nedd4-2 in dopamine transporter ubiquitination and endocytosis. $J$ Neurosci 26:8195-8205.

Staub O and Rotin D (2006) Role of ubiquitylation in cellular membrane transport. Physiol Rev 86: 669-707. 
Terada T and Inui K (2007) Gene expression and regulation of drug transporters in the intestine and kidney. Biochem Pharmacol 73:440-449.

Villar SR, Brandoni A, Anzai N, Endou H, and Torres AM (2005) Altered expression of rat renal cortical OAT1 and OAT3 in response to bilateral ureteral obstruction. Kidney Int 68:2704-2713.

Vina-Vilaseca A and Sorkin A (2010) Lysine 63-linked polyubiquitination of the dopamine transporter requires WW3 and WW4 domains of Nedd4-2 and UBE2D ubiquitin-conjugating enzymes. J Biol Chem 285:7645-7656.

Wang L and Sweet DH (2013) Renal organic anion transporters (SLC22 family): expression, regulation, roles in toxicity, and impact on injury and disease. AAPS J 15:53-69.

Xu D, Wang H, and You G (2016a) An essential role of Nedd4-2 in the ubiquitination, expression, and function of organic anion transporter-3. Mol Pharm 13:621-630.

Xu D, Wang H, Zhang Q, and You G (2016b) Nedd4-2 but not Nedd4-1 is critical for protein kinase C-regulated ubiquitination, expression, and transport activity of human organic anion transporter 1. Am J Physiol Renal Physiol 310:F821-F831.

You G (2002) Structure, function, and regulation of renal organic anion transporters. Med Res Rev 22:602-616.
Zhang Q, Hong M, Duan P, Pan Z, Ma J, and You G (2008) Organic anion transporter OAT1 undergoes constitutive and protein kinase C-regulated trafficking through a dynamin- and clathrin-dependent pathway. J Biol Chem 283:32570-32579.

Zhang Q, Li S, Patterson C, and You G (2013) Lysine 48-linked polyubiquitination of organic anion transporter-1 is essential for its protein kinase C-regulated endocytosis. Mol Pharmacol $\mathbf{8 3}$ 217-224.

Zhang W, Na T, Wu G, Jing H, and Peng JB (2010) Down-regulation of intestinal apical calcium entry channel TRPV6 by ubiquitin E3 ligase Nedd4-2. J Biol Chem 285: 36586-36596

Address correspondence to: Dr. Guofeng You, Department of Pharmaceutics, Rutgers University, 160 Frelinghuysen Road, Piscataway, NJ 08854. E-mail: gyou@ pharmacy.rutgers.edu 